\title{
Dynamics of the Self-Energy of the Gd(0001) Surface State Probed by Femtosecond Photoemission Spectroscopy
}

\author{
P. A. Loukakos, ${ }^{1}$ M. Lisowski, ${ }^{1}$ G. Bihlmayer, ${ }^{2}$ S. Blügel, ${ }^{2}$ M. Wolf, ${ }^{1}$ and U. Bovensiepen ${ }^{1, *}$ \\ ${ }^{1}$ Fachbereich Physik, Freie Universität Berlin, Arnimallee 14, 14195 Berlin-Dahlem, Germany \\ ${ }^{2}$ Institut für Festkörperforschung, Forschungszentrum Jülich, 52425 Jülich, Germany
}

(Received 6 July 2006; published 28 February 2007)

\begin{abstract}
Transient changes of the complex self-energy of the $5 d_{z^{2}}$ surface state on $\operatorname{Gd}(0001)$ after intense optical excitation are investigated by femtosecond time-resolved photoemission. We observe an ultrafast $(<100 \mathrm{fs})$ broadening of the linewidth due to $e-e$ scattering followed by a decrease of the binding energy due to thermal expansion of the lattice. In addition, we resolve a periodic breathing of the band structure which originates from a coherent phonon. An amplitude of $1 \mathrm{pm}$ is derived from the binding energy shift upon lattice displacement calculated by density functional theory.
\end{abstract}

PACS numbers: 78.47.+p, 73.20.At, 79.60.-i

In a many-body system such as a metallic ferromagnet, quasiparticles (QPs) such as electron-hole pairs, phonons, and magnons are excited at finite temperatures $T$. Because of their mutual interactions, these excitations exhibit finite lifetimes [1]. The screened electron-electron $(e-e)$ interaction leads to inelastic decay of hot electrons via electronhole pair creation. Scattering of electrons (or holes) with phonons or magnons (or vice versa) results in energy and momentum transfer between the electron, lattice, and spin degree of freedom [2-4]. The search for the microscopic interaction mechanisms governing these processes is a current topic of fundamental research. Such insight has high relevance for applications such as magnetic random access memory, which requires control of spin torque transfer from an electrical current to a macroscopic magnetic moment [5].

Because of these QP interactions, the spectral function measured in photoemission (PE) differs from the calculated single particle electronic band structure. The concept of the complex self-energy $[2,6]$ accounts for this difference, whereby the real part represents the binding energy $E_{B}$. The imaginary part defines the scattering rate measured in PE spectroscopy by the linewidth $\Gamma[7,8]$. Separation of $e-e$ and $e$-ph scattering has been obtained $[8,9]$ for surface states of metals from the quasilinear temperature dependence of $\Gamma$, which is determined by the $e$-ph interaction and allows determination of the mass enhancement parameter $\lambda$. In case of the $5 d_{z^{2}}$ surface state on ferromagnetic $\operatorname{Gd}(0001)$, the corresponding analysis includes also $e$-magnon scattering $[10,11]$.

Processes driven by hot electrons have been studied by femtosecond time-resolved two-photon photoemission. At solid interfaces, dynamical binding energy changes of excited states have been attributed to energy transfer to nuclear motion [12-14]. These processes are induced by excitations of single QP whereby excitonic interactions are effectively screened. By increasing the excitation density to about $10^{-2}$ carriers/atom, the electron distribution function is driven far out of equilibrium [15-17] which facil- itates also the investigation of the time evolution of occupied electronic states and the respective self-energy [18]. In these experiments, a nonequilibrium regime becomes accessible where a considerable fraction of conduction electrons participates in this collective excitation. Furthermore, ultrashort laser pulses allow a coherent excitation of phonon and magnon modes; i.e., these excitations present a well-defined phase with respect to the pump pulse $[19,20]$. In $\mathrm{TaS}_{2}$, the breathing of the electronic structure induced by such a coherent phonon excitation has been observed recently using time-resolved PE (TRPE) [21].

In this Letter, we study the time evolution of $E_{B}$ and $\Gamma$ for the occupied component of the $5 d_{z^{2}}$ surface state (SS) on the $\operatorname{Gd}(0001)$ surface. The optically excited nonequilibrium state is characterized by scattering processes investigated by the transient evolution of $\Gamma$. Upon absorption of an intense femtosecond laser pulse, $\Gamma$ increases 3 times, which is attributed to rapid $e-e$ (or $h-h$ ) scattering. During the first $400 \mathrm{fs}$, $e$-e scattering dominates over $e$-ph and $e$-magnon scattering. Simultaneously, $E_{B}$ shifts towards the Fermi level $E_{F}$ due to thermal expansion of the lattice. A superimposed oscillatory modulation of $E_{B}$ is observed arising from a coherent phonon-magnon mode as observed before in nonlinear optical pump-probe experiments [20]. First-principle calculations of $E_{B}$ as a function of lattice contraction allow an estimation of the coherent phonon amplitude to $10^{-2} \AA$.

The experimental setup combines a femtosecond laser system and an ultrahigh vacuum (UHV) chamber [16,17]. Epitaxial Gd(0001) films of $10 \mathrm{~nm}$ thickness are grown on $\mathrm{W}(110)$ in UHV [20]. Laser pulses at $h \nu_{\mathrm{IR}}=1.5 \mathrm{eV}$ with 55 fs duration and $4 \mu \mathrm{J}$ pulse energy are generated by an amplified Ti:sapphire laser system at a $300 \mathrm{kHz}$ repetition rate. A $3 \mu \mathrm{J}$ portion serves as a $s$-polarized pump pulse, and $1 \mu \mathrm{J}$ is used to generate the time-delayed $90 \mathrm{fs}$ $p$-polarized probe pulse at $h \nu_{\mathrm{UV}}=6.0 \mathrm{eV}$. Both are focused onto the Gd surface kept in UHV at $T=30 \mathrm{~K}$. In TRPE, absorption of pump laser pulses excites a nonequilibrium distribution of electrons and holes [15]. The time 
evolution of both the occupied and the normally unoccupied electronic structures is probed in direct photoemission induced by the time-delayed UV probe pulse since the photon energy $h \nu_{\mathrm{UV}}$ is above the work function $\Phi$ (inset in Fig. 1). According to Koopman's theorem, the measured kinetic energy of photoelectrons $E_{\text {kin }}$ determines $E_{B}=$ $h \nu_{\mathrm{UV}}-E_{\text {kin }}-\Phi$.

In Fig. 1, the PE intensity is shown as a function of $E_{B}$ and pump-probe delay $\Delta t$. Before excitation, the SS appears as a sharp feature at $E_{B}=180 \mathrm{meV}$. After the excitation $(\Delta t=0)$, hot electrons are observed for $E_{B}<$ 0 which are discussed in Ref. [17]. For $E_{B}>0$, the dominating feature is the occupied component of the exchangesplit SS. $E_{B}, \Gamma$, and the population of the state present pronounced changes with delay [22]. The intensity drop after excitation represents the generation of holes. An analysis of the area under the SS peak shows that the intensity is reduced up to $20 \%$ (not shown). This suggests an excitation of every fifth SS electron and emphasizes the intense optical excitation. For further analysis, we fit the spectra [Fig. 2(a)]. Based on our earlier investigations $[16,17]$, we consider a time-dependent electron distribution function $f(E, \Delta t)$ multiplied with a Lorentzian which determines $\Gamma$ and $E_{B}$. This product is fit to the spectra after convolution with the experimental resolution [23], which reproduces the spectra very well.

From this analysis, we obtain the transient evolution of $\Gamma$, which is depicted in Fig. 2(b). Remarkably, we find a threefold increase from $\Gamma=27 \mathrm{meV}$ to $\Gamma=86 \mathrm{meV}$ upon excitation. Subsequently, $\Gamma$ relaxes within 1 ps to an intermediate value of $57 \mathrm{meV}$. The recovery of the equilibrium value occurs within $130 \mathrm{ps}$ when the surface cools by heat

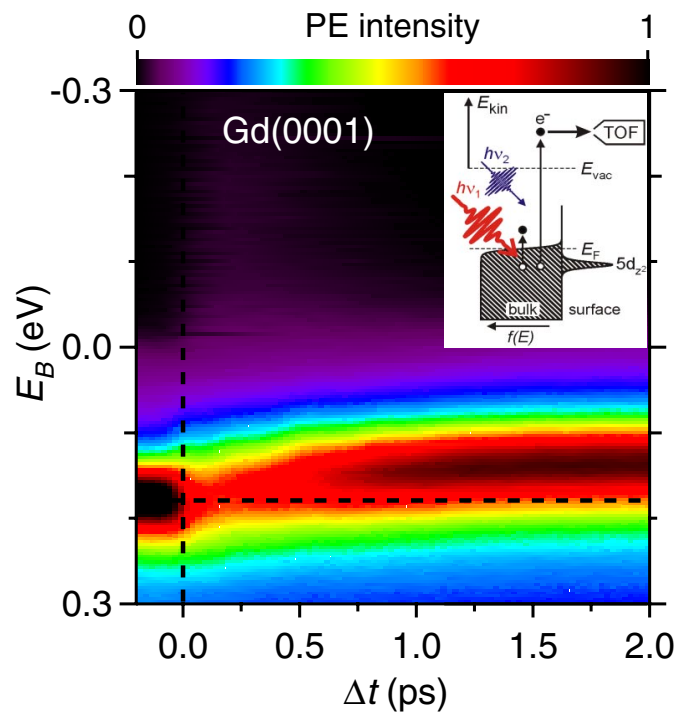

FIG. 1 (color online). False color representation of the TRPE intensity in normal emission as a function of binding energy $E_{B}$ and pump-probe delay $\Delta t$ for an absorbed IR fluence of $F=$ $1.0 \pm 0.1 \mathrm{~mJ} / \mathrm{cm}^{2}$. The dashed horizontal line denotes $E_{B}=$ $0.18 \mathrm{eV}$, which is the equilibrium value; the vertical one denotes $\Delta t=0$. The inset depicts TRPE schematically. dissipation. To understand the evolution during the first few picoseconds, we employ a description which combines a two-temperature model (TTM) [24] to obtain the transient electron and lattice temperatures $T_{e}$ and $T_{l}$ with analytical expressions for $\Gamma$ that account for $e-e$ and $e$-ph scattering. The TTM simplifies the processes after optical excitation as an instantaneous increase in $T_{e}$. Subsequently, $T_{e}$ is lowered due to $e$-ph scattering leading to a concomitant increase in $T_{l}$. After $200 \mathrm{fs}$, the electron system has thermalized, and $f(E, \Delta t)$ measured in TRPE [16,25] is a Fermi-Dirac distribution, i.e., $f(E, \Delta t)=f\left(E, T_{e}\right)$, and the TTM is valid. The TTM allows determination of $T_{l}$, which increases to $150 \mathrm{~K}$ within $2 \mathrm{ps,}$, when $T_{e}=T_{l}$. Since the electrons are described by a thermalized $f(E)$, we assume that equilibrium concepts for $\Gamma$ hold in combination with Matthiesen's rule $\Gamma=\Gamma_{e-e}+\Gamma_{e-\mathrm{ph} / m}$. However, extraction of $T_{e}$ from TRPE at $\Delta t<200 \mathrm{fs}$ underestimates the energy density, and at these early delays our analysis represents a lower limit for scattering rates. Following [26-28], we use

$$
\begin{gathered}
\Gamma_{e-e}=\beta\left[\left(\pi k_{B} T_{e}\right)^{2}+\left(E-E_{F}\right)^{2}\right], \\
\Gamma_{e-\mathrm{ph} / m}=\frac{2 \pi}{\left(\hbar \omega_{D}\right)^{2}} \lambda \int_{0}^{\hbar \omega_{D}} \epsilon^{2} A\left(\epsilon, T_{e}, T_{l}\right) d \epsilon .
\end{gathered}
$$

Here $A=\left[1-f\left(E-\epsilon, T_{e}\right)+2 n\left(\epsilon, T_{l}\right)+f\left(E+\epsilon, T_{e}\right)\right], n$ is the Boltzmann distribution, $\omega_{D}=14 \mathrm{meV} / \hbar$ is the Debye frequency, and $\Gamma_{e-\mathrm{ph} / m}$ comprises $e$-ph and $e$-magnon scattering. Alternatively, one could consider a three-temperature model including a spin temperature [29] and a separate $\Gamma_{e-m}$. We use an effective $\Gamma_{e-\mathrm{ph} / m}$ because (i) it is unclear how $e$-magnon scattering is to be included explicitly, and (ii) we focus on the initial dynamics where $e-e$ scattering dominates. For the simulation of $\Gamma$ in Fig. 2(b), the transient $T_{e}$ and $T_{l}$, as determined by the TTM including the specific heat of phonons and magnons, are used as an input. The experimental result for $\Gamma$ is
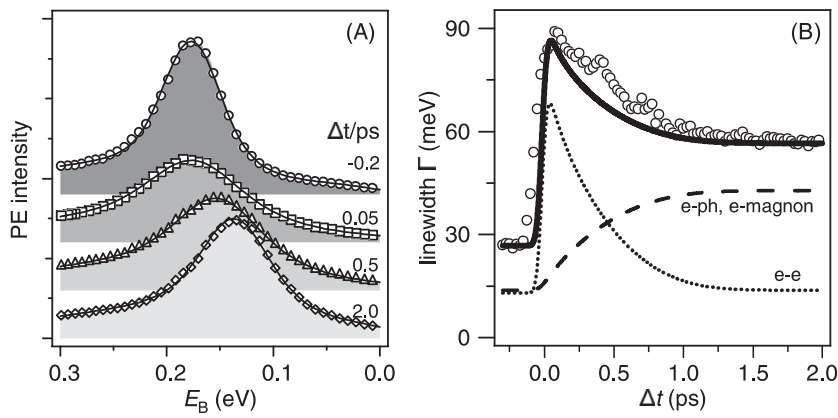

FIG. 2. (a) TRPE spectra at indicated time delays with fits (solid lines). (b) The time evolution of the linewidth $\Gamma$ of the occupied SS component $(O)$. The accuracy of the fitting analysis is about $\pm 5 \mathrm{meV}$. The solid line represents a model description based on the TTM with two contributions: (i) $e-e$ scattering (dotted curve) and (ii) $e$-magnon and $e$-phonon scattering (dashed curve). The equilibrium temperature is $30 \mathrm{~K}$. 
reproduced fairly well, and $\Gamma_{e-e}$ and $\Gamma_{e-\mathrm{ph} / m}$ can be separated. We find that for $\Delta t<400$ fs $e-e$ scattering dominates over $\Gamma_{e-\mathrm{ph} / m}$, which is reasonable due to the high nonequilibrium carrier density after optical excitation. Such a regime is not accessible in equilibrium [7,9$11,26,28]$, which highlights the potential of TRPE to obtain insight into transient processes. $\Gamma_{e-\mathrm{ph} / m}$ takes over at later delays and originates from energy transfer to the lattice and spin system. The determined value $\beta=0.2 \mathrm{eV}^{-1}$ agrees with Ref. [28]. Our result $\lambda=0.45$ is 2 times smaller compared to experiments performed in equilibrium $[10,28]$ but is in agreement with calculations [30]. Since our linewidth is smaller than reported in Refs. [10,28] for $30 \mathrm{~K}$, we consider defect-induced scattering as an origin of this deviation [8]. In the following, we analyze $E_{B}$ and benefit from the analysis of $\Gamma$ as a characterization of the photoexcited state.

Figure 3(a) shows the time dependence of $E_{B}$. On top of a continuous shift towards $E_{F}$ (solid line), we find an oscillating contribution. As shown above, electrons are in equilibrium with phonons and magnons after 2 ps. Thus, the continuous change of $E_{B}$ over 2 ps can, in principle, occur due to phonons or magnons. Our earlier study [17] has shown that the exchange splitting $\Delta_{\text {ex }}$ remains unaffected under such nonequilibrium conditions. Thus, we conclude that the reduction in $E_{B}$ is dominated by thermal expansion. Subtraction of the incoherent background from $E_{B}$ reveals the coherent component $\delta E_{B}$ [Fig. 3(b)]. After $250 \mathrm{fs}$, we resolve a damped oscillating behavior at a frequency of $3.0(1) \mathrm{THz}$ with a damping time of $1.0(1) \mathrm{ps}$ as determined by a fit (solid line). Frequency and damping are identical with the ones of the coherent

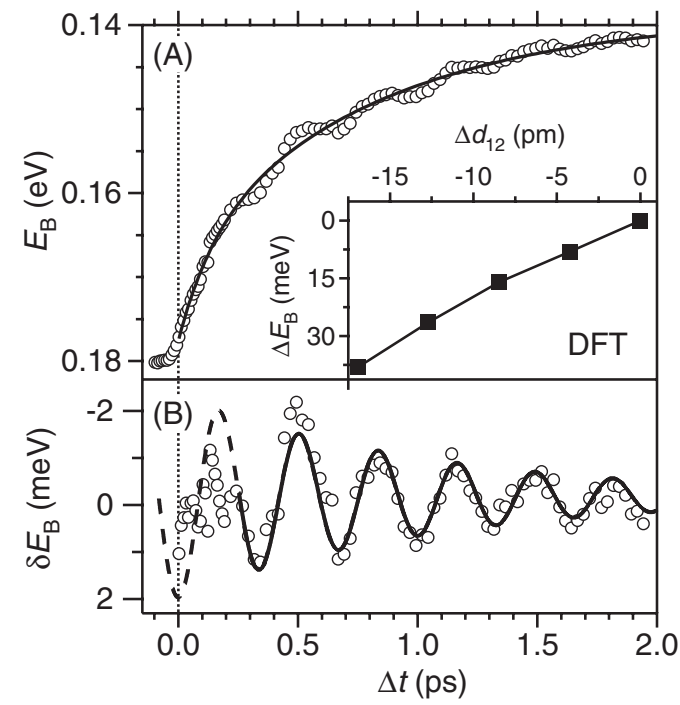

FIG. 3. (a) Time evolution of $E_{B}$ with a coherent part on an incoherent thermal background (solid line). Inset: $E_{B}$ increase upon contraction of $d_{12}$ as calculated by DFT. Note that $E_{B}$ increases from top to bottom. (b) The oscillatory component. The solid line is a fit by an exponentially damped cosine; the dashed line shows its extrapolation to time zero. phonon-magnon mode observed with nonlinear optics on the $\operatorname{Gd}(0001)$ surface [20]. This surface mode is derived from a LO phonon where the [0001] planes vibrate against each other. At the surface, the surface plane vibrates with respect to the bulk, and the interlayer distance at the surface $d_{12}$ is modulated by $\delta$. The mode is excited by a pump-induced redistribution of electrons and spins between surface and bulk states [25]. As a consequence, the minimum in the excited state potential $d_{12}^{*}$ is displaced by $\delta$ with respect to $d_{12}^{0}$ for the ground state [19] as illustrated in Fig. 4, right. Since a change in $d_{12}$ influences $E_{B}$, we conclude that the system oscillates along $d_{12}$ on the optically excited potential surface. The driving force in the coherent phonon excitation is given by the gradient of the potential, and, subsequently, energy is redistributed periodically between the potential and kinetic energy. Since PE probes the electron subsystem, we monitor the adiabatic breathing of $E_{B}$ that originates from the modulation of $d_{12}$ (Fig. 4, right). In contrast, the elementary processes of $e-e$, $e$-ph, and $e$-magnon scattering lead to transient broadening of $\Gamma$ (Fig. 4, left).

To estimate the phonon amplitude $\delta$, we calculate $E_{B}$ as a function of $d_{12}$ by density functional theory (DFT). We cannot describe the nonequilibrium state explicitly and do not expect that anharmonic effects such as thermal expansion are reproduced. On the other hand, small harmonic variations of $d_{12}$ will be included. The generalized gradient approximation was employed [31] in combination with the full-potential linearized augmented plane wave method in film geometry [32] as implemented in the FLEUR code. The $4 f$ electrons are included in the $4 f$-core method [33]. The Gd surface was simulated by a relaxed 10-layer film embedded in semi-infinite vacua. For the calculations, a plane wave cutoff of $k_{\max }=3.8$ a.u. $^{-1}$ was used, and the irreducible part of the two-dimensional Brillouin zone was sampled with 21 special $k$ points.

The calculated $E_{B}$ change upon contraction of $d_{12}$ is depicted in Fig. 3, and we estimate from $\delta E_{B, 0}=2 \mathrm{meV}$ a lattice displacement of $\delta=1 \mathrm{pm}$ or $3 \times 10^{-3} d_{12}$ [34]. This is a small, but reasonable, value for the employed excitation conditions and corroborates the high sensitivity

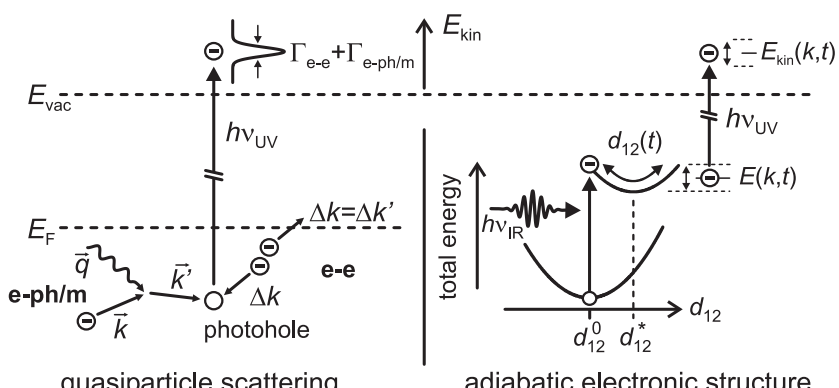

FIG. 4. Processes leading to transient changes in photoemission. Left: QP scattering due to relaxation of the excited state. Right: Periodic change of $E_{B}$ due to the coherent phonon along $d_{12}$. 
of TRPE. Time-resolved $x$-ray diffraction would probe the lattice dynamics directly [35,36]. However, in homogenous solids available setups are not sensitive enough for such small amplitudes and lack surface sensitivity.

Finally, we discuss the effects of electron-phonon interaction, which we resolve in changes of the linewidth and binding energy (see Fig. 4). The $e$-ph contribution to $\Gamma$ is described by $\lambda$ and deformation potential scattering of electrons $[2,26,28]$. In our time-resolved experiment, we succeeded to monitor the "breathing" of the electronic structure during a lattice vibration by exciting a coherent phonon with femtosecond laser pulses. Under equilibrium conditions, the effect of lattice vibration on $E_{B}$ cannot be resolved owing to the uncorrelated thermal motion of individual phonons and similar considerations as for the Debye-Waller factor. Using the above estimated real space amplitude of the phonon, we compare the population of the $3 \mathrm{THz}$ mode for the nonequilibrium coherent excitation $N_{\text {neq }}$ with the population of the mode in thermal equilibrium $N_{\text {eq }}$. We calculate $N_{\text {neq }}$ from a harmonic potential $U=m \Omega^{2} \delta^{2} / 2=0.3 \mathrm{meV}$, where $m$ is the atomic mass of $\mathrm{Gd}, \Omega /(2 \pi)=3 \mathrm{THz}$, and $\delta=1 \mathrm{pm}$. Since $\hbar \Omega=$ $12 \mathrm{meV}$, we find $N_{\text {neq }}=0.025$. At early delays when $\delta=$ $1 \mathrm{pm}$, the energy resides in the electronic subsystem and $T_{l}=30 \mathrm{~K}$. The corresponding thermal population is $n\left(\Omega, T_{l}\right)=9 \times 10^{-3}$, and we quantify the excess population in the nonequilibrium coherent phonon state $N_{\text {neq }} / N_{\text {eq }}=2.8$. For larger delay, $T_{l}$ increases, resulting in more frequent scattering between coherent and thermal phonons. This leads - in combination with ph-e scattering - to damping of the coherent mode during several picoseconds as observed in Fig. 3.

In summary, we have investigated the ultrafast dynamics of the complex self-energy for the $\operatorname{Gd}(0001) 5 d_{z^{2}}$ surface state. Using time-resolved photoemission, we observe a threefold quasi-instantaneous increase in the linewidth which originates from $e-e$ scattering. The binding energy exhibits a periodic change at a frequency of $3 \mathrm{THz}$ due to a coherent phonon motion. Such breathing of the electronic structure upon lattice vibration can be studied only under nonequilibrium conditions. Employing DFT calculations to estimate the phonon amplitude, we determine the population of the coherent phonon to be 3 times enhanced compared to equilibrium conditions.

Funding by the DFG (SPP 1133) and by the EU is gratefully acknowledged.

*Electronic addresses: uwe.bovensiepen@physik.fuberlin.de

http://www.physik.fu-berlin.de/ bovensie

[1] D. Pines, Elementary Excitations in Solids (Benjamin, New York, 1964).

[2] P. M. Echenique et al., Surf. Sci. Rep. 52, 219 (2004).

[3] M. Plihal and D. L. Mills, Phys. Rev. B 58, 14407 (1998).
[4] V.P. Zhukov, E. V. Chulkov, and P. M. Echenique, Phys. Rev. Lett. 93, 096401 (2004).

[5] J. C. Slonczewski, J. Magn. Magn. Mater. 159, L1 (1996).

[6] I.-W. Lyo and E. W. Plummer, Phys. Rev. Lett. 60, 1558 (1988).

[7] J. J. Paggel et al., Phys. Rev. Lett. 83, 1415 (1999).

[8] F. Reinert, G. Nicolay, S. Schmidt, D. Ehm, and S. Hüfner, Phys. Rev. B 63, 115415 (2001).

[9] T. Valla, A. V. Fedorov, P. D. Johnson, and S. L. Hulbert, Phys. Rev. Lett. 83, 2085 (1999).

[10] A. V. Fedorov et al., Phys. Rev. B 65, 212409 (2002).

[11] D. Wegner, A. Bauer, and G. Kaindl, Phys. Rev. B 73, 165415 (2006).

[12] C. Gahl et al., Phys. Rev. Lett. 89, 107402 (2002).

[13] A. D. Miller et al., Science 297, 1163 (2002).

[14] B. Li, J. Zhao, K. Onda, K. D. Jordan, J. Yang, and H. Petek, Science 311, 1436 (2006).

[15] W. S. Fann et al., Phys. Rev. Lett. 68, 2834 (1992).

[16] M. Lisowski et al., Appl. Phys. A 78, 165 (2004).

[17] M. Lisowski et al., Phys. Rev. Lett. 95, 137402 (2005).

[18] H.-S. Rhie, H. A. Dürr, and W. Eberhard, Appl. Phys. A 82, 9 (2006).

[19] T. Dekorsy, G. C. Cho, and H. Kurz, Coherent Phonons in Condensed Media, Topics in Applied Physics Vol. 76 (Springer, New York, 2000).

[20] A. Melnikov et al., Phys. Rev. Lett. 91, 227403 (2003).

[21] L. Perfetti et al., Phys. Rev. Lett. 97, 067402 (2006).

[22] Because of the experimental conditions, space charge effects are independent on pump-probe delay and have been quantified by taking spectra as a function of laser fluence. Their contributions are at maximum $5 \mathrm{meV}$ for $\Gamma$ and $10 \mathrm{meV}$ for $E_{B}$.

[23] The experimental resolution has two contributions. The resolution of the time-of-flight spectrometer is $<20 \mathrm{meV}$ for the kinetic energies analyzed here. The bandwidth of the ultrashort laser pulses is $30 \mathrm{meV}$.

[24] S. I. Anisimov et al., Sov. Phys. JETP 39, 375 (1974).

[25] U. Bovensiepen, J. Phys. Condens. Matter (to be published).

[26] R. Matzdorf, Surf. Sci. Rep. 30, 153 (1998).

[27] G. Grimval, The Electron-Phonon Interaction in Metals (North-Holland, Amsterdam, 1981).

[28] A. Rehbein et al., Phys. Rev. B 67, 033403 (2003).

[29] E. Beaurepaire et al., Phys. Rev. Lett. 76, 4250 (1996).

[30] H. L. Skriver and I. Mertig, Phys. Rev. B 41, 6553 (1990).

[31] J. P. Perdew et al., Phys. Rev. Lett. 77, 3865 (1996).

[32] E. Wimmer et al., Phys. Rev. B 24, 864 (1981).

[33] Ph. Kurz et al., J. Phys. Condens. Matter 14, 6353 (2002).

[34] Such a small phonon amplitude does not explain the magnon contribution of the coherent phonon-magnon mode by the proposed periodic modulation of $\Delta_{\mathrm{ex}}$ [20]. Additional DFT calculations for the unoccupied SS show that both SS components exhibit the same increase in $\left|E_{B}\right|$ with reducing $d_{12}$. Thus, no change in $\Delta_{\mathrm{ex}}$ is observed. We conclude that, due to the localized character of the $5 d_{z^{2}}$ $\mathrm{SS}$, changes in $\Delta_{\mathrm{ex}}$ do not dominate, but local spin flip scattering [17] is responsible.

[35] K. Sokolowski-Tinten et al., Nature (London) 422, 287 (2003).

[36] M. Bargheer et al., Science 306, 1771 (2004). 